\title{
Supporting Information: Simulation-based Superstructure Optimization for Process Synthesis of Aromatics Production from Methanol
}

\author{
Dan Zhang ${ }^{\dagger}$, Jianrong Jiang ${ }^{\dagger}$, Minbo Yang ${ }^{\dagger, *}$, Xiao Feng ${ }^{\dagger}$, Yufei Wang \\ $\dagger$ Shaanxi Key Laboratory of Energy Chemical Process Intensification, School of Chemical \\ Engineering and Technology, Xi'an Jiaotong University, No.28, Xianning West Road, Beilin \\ District, Xi'an, Shaanxi 710049, China \\ * State Key Laboratory of Heavy Oil Processing, China University of Petroleum (Beijing), No.18, \\ Fuxue Road, Changping District, Beijing 102249, China \\ *Corresponding author: yangmb@mail.xjtu.edu.cn
}

The number of pages: 11

The number of figures: 1

The number of tables: 12

\section{Contents:}

Figure S1: S matrix for the product separation unit

Table S1: The product distributions of the four different reactors

Table S2: The product specifications

Table S3: Operating parameters for reactors

Table S4: Outlet pressure of each pump or compressor

Table S5: Outlet temperature of each cooler/heater

Table S6: Operating parameters of distillation columns used in the process simulation

Table S7: Input parameters for energy consumption/economic evaluation

Table S8: Bare-module factors of the equipment and process machinery

Table S9: Base cost of key equipment

Table S10: Input parameters for annual sales

Table S11: Input parameters for total annual production costs

Table S12: The characterization factors 


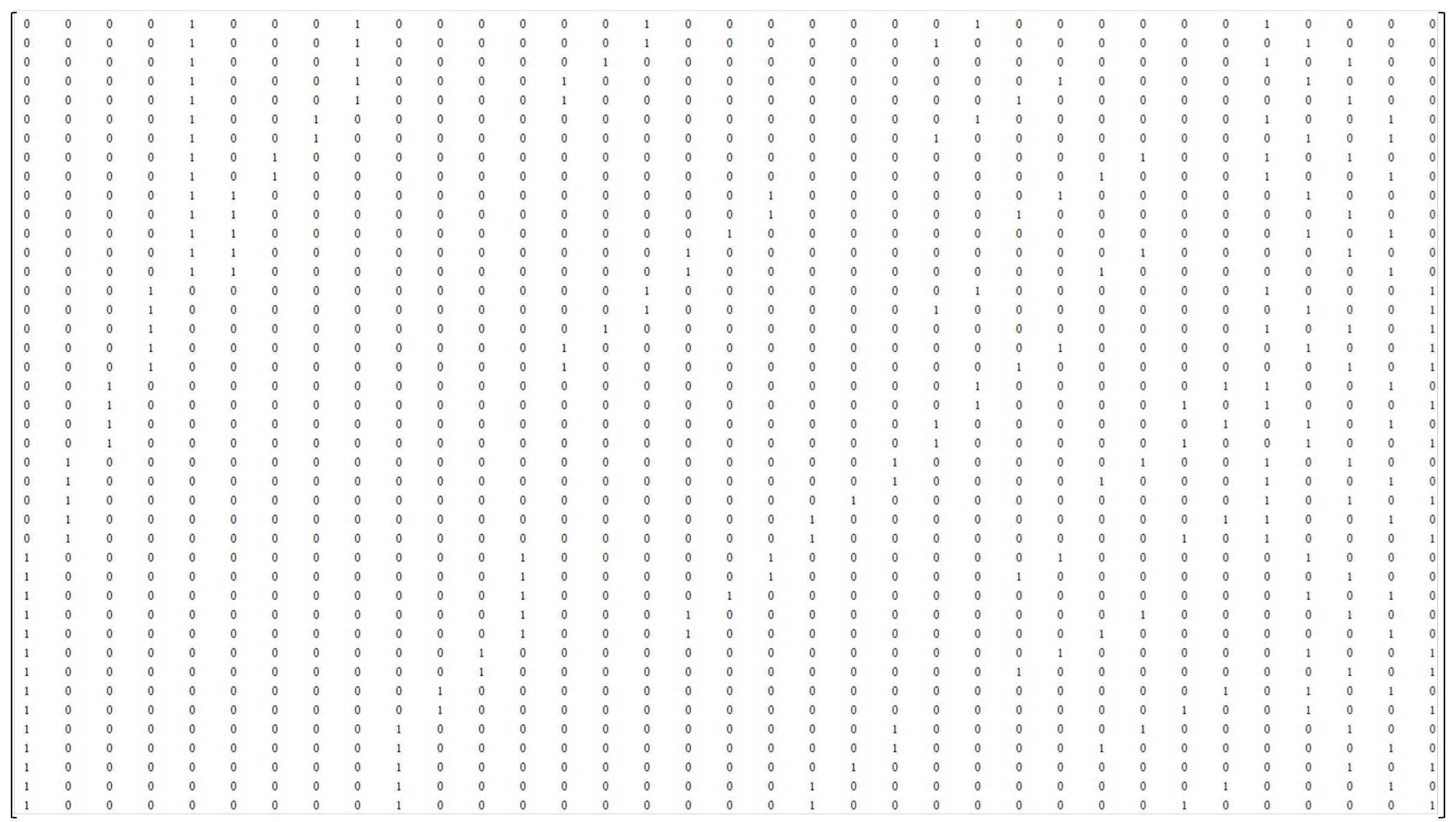

Figure S1. S matrix of the product separation unit. 
Table S1. The product distributions of the four different reactors.

\begin{tabular}{|c|c|c|c|c|c|}
\hline \multirow[b]{2}{*}{ No. } & \multirow[b]{2}{*}{ Component } & \multicolumn{4}{|c|}{ Mass fraction $/ \mathrm{wt} \%$} \\
\hline & & $\mathrm{SSFB}^{1}$ & TSFB $^{1}$ & THSFB- $1^{2}$ & THSFB- $2^{3}$ \\
\hline 1 & $\mathrm{H}_{2}$ & 0.41 & 0.37 & 0.37 & 0.37 \\
\hline 2 & $\mathrm{CO}$ & 0.49 & 0.32 & 0.32 & 0.32 \\
\hline 3 & $\mathrm{CH}_{4}$ & 1.60 & 1.83 & 2.00 & 3.53 \\
\hline 4 & $\mathrm{C}_{2} \mathrm{H}_{4}$ & 0.99 & 0.94 & 0.19 & 0.71 \\
\hline 5 & $\mathrm{C}_{2} \mathrm{H}_{6}$ & 1.15 & 1.38 & 1.52 & 2.31 \\
\hline 6 & $\mathrm{CO}_{2}$ & 0.05 & 0.09 & 0.09 & 0.08 \\
\hline 7 & $\mathrm{C}_{3} \mathrm{H}_{6}$ & 1.44 & 1.36 & 0.13 & 0.47 \\
\hline 8 & $\mathrm{C}_{3} \mathrm{H}_{8}$ & 16.56 & 13.10 & 8.37 & 6.83 \\
\hline 9 & $1-\mathrm{C}_{4} \mathrm{H}_{8}$ & 0.52 & 0.50 & 0.11 & 0.24 \\
\hline 10 & $n-C_{4} H_{10}$ & 4.03 & 4.68 & 2.71 & 1.46 \\
\hline 11 & $\mathrm{n}-\mathrm{C}_{5} \mathrm{H}_{12}$ & 3.10 & 3.89 & 0.95 & 0.42 \\
\hline 12 & $\mathrm{C}_{6} \mathrm{H}_{6}$ & 0.62 & 1.54 & 2.38 & 4.62 \\
\hline 13 & $\mathrm{C}_{7} \mathrm{H}_{8}$ & 3.65 & 4.93 & 8.56 & 11.31 \\
\hline 14 & E-Benzene & 0.14 & 0.25 & 0.62 & 0.42 \\
\hline 15 & PX & 2.55 & 2.33 & 2.95 & 2.41 \\
\hline 16 & MX & 5.12 & 5.14 & 6.42 & 5.23 \\
\hline 17 & OX & 2.41 & 2.37 & 3.47 & 2.82 \\
\hline 18 & 1M4-Ebenzene & 0.75 & 0.71 & 1.67 & 1.07 \\
\hline 19 & 135-Mbenzene & 0.75 & 0.71 & 1.67 & 1.07 \\
\hline 20 & 124-Mbenzene & 0.75 & 0.71 & 1.67 & 1.07 \\
\hline 21 & 123-MBenzene & 0.75 & 0.71 & 1.67 & 1.07 \\
\hline 22 & $\mathrm{H}_{2} \mathrm{O}$ & 52.16 & 52.16 & 52.16 & 52.16 \\
\hline
\end{tabular}

Table S2. The product specifications.

\begin{tabular}{|c|c|c|c|c|c|c|}
\hline & \multicolumn{6}{|c|}{ Products } \\
\hline & Benzene & Toluene & Xylene & $\begin{array}{c}\mathrm{C}_{9}+ \\
\text { aromatics }\end{array}$ & & Dry gas \\
\hline Items & & Specifica & ons $/ \mathrm{wt} \%{ }^{4}$ & & Items & Specifications $/$ vol $^{5}{ }^{5}$ \\
\hline Benzene & & $<0.05$ & & & & \\
\hline Toluene & 0.05 & & $<0.10$ & & & \\
\hline Xylene & & $<0.10$ & & $<0.05$ & $\mathrm{C}_{3}+$ & $<=3$ \\
\hline $\begin{array}{c}\text { Non- } \\
\text { aromatics }\end{array}$ & $<0.10$ & $<0.25$ & $<0.25$ & & & \\
\hline
\end{tabular}


Table S3. Operating parameters for reactors

\begin{tabular}{ccc}
\hline Reactor & Temperature $/{ }^{\circ} \mathrm{C}$ & Pressure $/ \mathrm{kPa}$ \\
\hline Methanol aromatization & 475 & 400 \\
Light hydrocarbon aromatization $^{\text {a }}$ & 500 & 120 \\
\hline
\end{tabular}

${ }^{\text {a }}$ To simplify the model, the reactor volumes are fixed as $133 \mathrm{~m}^{3}$, which makes the light hydrocarbon conversions reach to $75 \%$ in the 42 production routes with SSFB reactor.

Table S4. Outlet pressure of each pump or compressor

\begin{tabular}{ccc}
\hline Item & Equipment number & Outlet pressure/kPa \\
\hline \multirow{2}{*}{ Pump } & P-100 & 400 \\
& P-101 & 150 \\
\hline K-100 & 150 \\
& K-101 & 1800 \\
K-102 & 650 \\
K-103 & 150 \\
& K-104 & 150 \\
K-105 & 1800 \\
K-106 & 650 \\
& K-107 & 150 \\
& K-108 & 1800 \\
& K-109 & 650 \\
& K-110 & 650 \\
& K-111 & 150 \\
& K-112 & 1800 \\
& K-113 & 150 \\
\hline
\end{tabular}

Table S5. Outlet temperature of each cooler/heater

\begin{tabular}{cc}
\hline Equipment number & Outlet temperature $/{ }^{\circ} \mathrm{C}$ \\
\hline E-101 & 30 \\
E-102 & 30 \\
Fired heaters & 630 \\
\hline
\end{tabular}


Table S6. Operating parameters of distillation columns used in the process simulation. (In pressure cell, the first value represents the operating pressure at the top of a column, and the second value represents the operating pressure at the bottom of a column.)

\begin{tabular}{|c|c|c|c|c|}
\hline Column & Number of trays & Inlet stage & Reflux ratio & pressure $/ \mathrm{kPa}$ \\
\hline $\mathrm{T}-100$ & 30 & 15 & 2.36 & {$[1700,1800]$} \\
\hline $\mathrm{T}-101$ & 20 & 7 & 0.30 & {$[600,650]$} \\
\hline $\mathrm{T}-102$ & 36 & 25 & 0.17 & {$[120,150]$} \\
\hline $\mathrm{T}-103$ & 35 & 21 & 0.11 & {$[120,150]$} \\
\hline $\mathrm{T}-104$ & 35 & 28 & 1.15 & {$[120,150]$} \\
\hline $\mathrm{T}-105$ & 30 & 15 & 2.5 & {$[1700,1800]$} \\
\hline $\mathrm{T}-106$ & 20 & 7 & 0.76 & {$[600,650]$} \\
\hline $\mathrm{T}-107$ & 36 & 25 & 0.31 & {$[120,150]$} \\
\hline T-108 & 35 & 21 & 0.19 & {$[120,150]$} \\
\hline T-109 & 20 & 7 & 6.46 & {$[600,650]$} \\
\hline $\mathrm{T}-110$ & 36 & 25 & 0.25 & {$[120,150]$} \\
\hline $\mathrm{T}-111$ & 35 & 21 & 0.22 & {$[120,150]$} \\
\hline $\mathrm{T}-112$ & 35 & 28 & 3.03 & {$[120,150]$} \\
\hline $\mathrm{T}-113$ & 30 & 15 & 2.36 & {$[1700,1800]$} \\
\hline $\mathrm{T}-114$ & 20 & 7 & 0.49 & {$[600,650]$} \\
\hline $\mathrm{T}-115$ & 36 & 25 & 0.20 & {$[120,150]$} \\
\hline $\mathrm{T}-116$ & 20 & 7 & 4.94 & {$[600,650]$} \\
\hline $\mathrm{T}-117$ & 36 & 25 & 0.23 & {$[120,150]$} \\
\hline $\mathrm{T}-118$ & 35 & 21 & 0.21 & {$[120,150]$} \\
\hline $\mathrm{T}-119$ & 36 & 25 & 26.39 & {$[120,150]$} \\
\hline $\mathrm{T}-120$ & 35 & 21 & 2.83 & {$[120,150]$} \\
\hline $\mathrm{T}-121$ & 35 & 28 & 13.27 & {$[120,150]$} \\
\hline $\mathrm{T}-122$ & 30 & 15 & 2.36 & {$[1700,1800]$} \\
\hline $\mathrm{T}-123$ & 20 & 7 & 0.18 & {$[600,650]$} \\
\hline $\mathrm{T}-124$ & 20 & 7 & 3.21 & {$[600,650]$} \\
\hline $\mathrm{T}-125$ & 36 & 25 & 0.56 & {$[120,150]$} \\
\hline $\mathrm{T}-126$ & 36 & 25 & 24.89 & {$[120,150]$} \\
\hline $\mathrm{T}-127$ & 35 & 21 & 2.63 & {$[120,150]$} \\
\hline $\mathrm{T}-128$ & 35 & 21 & 2.73 & {$[120,150]$} \\
\hline $\mathrm{T}-129$ & 35 & 28 & 14.29 & {$[120,150]$} \\
\hline $\mathrm{T}-130$ & 30 & 15 & 2.36 & {$[1700,1800]$} \\
\hline $\mathrm{T}-131$ & 20 & 7 & 0.42 & {$[600,650]$} \\
\hline $\mathrm{T}-132$ & 36 & 25 & 21.61 & {$[120,150]$} \\
\hline $\mathrm{T}-133$ & 35 & 21 & 2.59 & {$[120,150]$} \\
\hline $\mathrm{T}-134$ & 35 & 28 & 29.94 & {$[120,150]$} \\
\hline
\end{tabular}


Table S7. Input parameters for energy consumption/economic evaluation.

\begin{tabular}{ccccc}
\hline Item & Value & Unit & Note & Sources \\
\hline$\phi_{\text {in }}$ & $1038.68 / 944.53 / 567.15 / 561.65$ & $\mathrm{~mol} / \mathrm{s}$ & $\mathrm{a}$ & - \\
$C_{a}$ & 53.71 & $\mathrm{~kJ} /(\mathrm{kmol} \cdot \mathrm{K})$ & - & - \\
$T_{\text {in }}$ & 303.15 & $\mathrm{~K}$ & - & - \\
$R$ & 8.314 & $\mathrm{~kJ} /(\mathrm{kmol} \cdot \mathrm{K})$ & - & - \\
& & & & \\
$\gamma$ & $15.65 / 5.65 / 1.30$ & - & $\mathrm{b}$ & - \\
$\xi_{\mathrm{c}}$ & $75 \%$ & - & - & - \\
$F$ & $815.91 / 874.31 / 1192.48 / 1112.6$ & $\mathrm{~L} / \mathrm{min}$ & $\mathrm{c}$ & - \\
& 8 & & & \\
$H_{\mathrm{P}}$ & $3.5 / 51.5 / 136.5$ & $\mathrm{~m}$ & $\mathrm{~d}$ & - \\
$\rho$ & 848.4 & $\mathrm{~g} / \mathrm{L}$ & - & - \\
$F_{\text {in }}$ & 15808.4 & $\mathrm{~L} / \mathrm{h}$ & - & - \\
$v_{\mathrm{s}}$ & 0.4 & $1 / \mathrm{h}$ & - & - \\
$F_{\mathrm{M}}$ & 1.4 & - & - & - \\
$P_{\mathrm{F}}$ & 18.69 & $\mathrm{kPaG}$ & - & - \\
$\beta$ & 0.6 & - & - & 6 \\
$H_{\mathrm{r}}$ & 7919.04 & $\mathrm{~h}$ & - & - \\
\hline SSFB-based & 7.822 & $10^{5} \mathrm{~kJ} / \mathrm{kmole}$ & - & - \\
TSFB-based & 7.893 & $10^{5} \mathrm{~kJ} / \mathrm{kmole}$ & - & - \\
THSFB-1-based & 7.124 & $10^{5} \mathrm{~kJ} / \mathrm{kmole}$ & - & - \\
THSFB-2-based & 7.465 & $10^{5} \mathrm{~kJ} / \mathrm{kmole}$ & - & - \\
\hline$\eta$ & 0.6 & - & - & - \\
$T R$ & $40 \%$ & - & - & 6 \\
$r$ & $10 \%$ & $\mathrm{yr}$ & - & - \\
\hline$T T$ & 20 & - & 7 \\
\hline & & & - & -
\end{tabular}

${ }^{a}$ There are different values when different methanol aromatization reactors are chosen.

${ }^{\mathrm{b}}$ There are different values when different product separation sequences are chosen.

${ }^{\mathrm{c}}$ There are different values when different methanol aromatization reactors are chosen.

${ }^{\mathrm{d}}$ There are different values when different product separation sequences are chosen.

Table S8. Bare-module factors of the equipment and process machinery.

\begin{tabular}{cc}
\hline Equipment & Bare-module factor $^{6}$ \\
\hline Heat exchanger/Heater & 3.17 \\
Cooler & 2.17 \\
Distillation column/Reactor & 4.16 \\
Pump & 3.30 \\
Compressor & 2.15 \\
\hline
\end{tabular}


Table S9. Base cost of key equipment.

\begin{tabular}{|c|c|c|}
\hline Item & Equipment number & Base cast $/ 10^{6} \$$ \\
\hline \multirow{35}{*}{ Distillation column } & $\mathrm{T}-100$ & 0.80 \\
\hline & $\mathrm{T}-101$ & 0.87 \\
\hline & $\mathrm{T}-102$ & 1.78 \\
\hline & $\mathrm{T}-103$ & 2.24 \\
\hline & $\mathrm{T}-104$ & 2.66 \\
\hline & $\mathrm{T}-105$ & 0.75 \\
\hline & T-106 & 0.91 \\
\hline & $\mathrm{T}-107$ & 2.00 \\
\hline & $\mathrm{T}-108$ & 2.23 \\
\hline & T-109 & 1.14 \\
\hline & $\mathrm{T}-110$ & 7.09 \\
\hline & $\mathrm{T}-111$ & 1.28 \\
\hline & $\mathrm{T}-112$ & 1.92 \\
\hline & $\mathrm{T}-113$ & 0.75 \\
\hline & $\mathrm{T}-114$ & 0.73 \\
\hline & $\mathrm{T}-115$ & 1.73 \\
\hline & $\mathrm{T}-116$ & 0.96 \\
\hline & $\mathrm{T}-117$ & 0.70 \\
\hline & $\mathrm{T}-118$ & 1.10 \\
\hline & $\mathrm{T}-119$ & 0.46 \\
\hline & $\mathrm{T}-120$ & 0.70 \\
\hline & $\mathrm{T}-121$ & 1.64 \\
\hline & $\mathrm{T}-122$ & 0.83 \\
\hline & $\mathrm{T}-123$ & 0.56 \\
\hline & $\mathrm{T}-124$ & 0.63 \\
\hline & $\mathrm{T}-125$ & 0.85 \\
\hline & $\mathrm{T}-126$ & 0.45 \\
\hline & $\mathrm{T}-127$ & 0.56 \\
\hline & $\mathrm{T}-128$ & 0.71 \\
\hline & $\mathrm{T}-129$ & 1.64 \\
\hline & $\mathrm{T}-130$ & 0.84 \\
\hline & $\mathrm{T}-131$ & 0.47 \\
\hline & $\mathrm{T}-132$ & 0.38 \\
\hline & $\mathrm{T}-133$ & 0.56 \\
\hline & $\mathrm{T}-134$ & 1.56 \\
\hline \multirow[b]{2}{*}{ Reactor } & Methanol aromatization & 1.99 \\
\hline & $\begin{array}{l}\text { Light hydrocarbon } \\
\text { aromatization }\end{array}$ & 0.74 \\
\hline Pump & P-100 & 0.02 \\
\hline \multirow{3}{*}{ Compressor } & K-101 & 5.83 \\
\hline & K-102 & 3.82 \\
\hline & K-103 & 0.71 \\
\hline
\end{tabular}




\begin{tabular}{ccc}
\hline K-104 & 0.57 \\
K-105 & 5.31 \\
K-106 & 3.46 \\
K-107 & 0.63 \\
K-108 & 4.46 \\
K-109 & 2.88 \\
K-110 & 1.65 \\
K-111 & 0.32 \\
K-112 & 2.00 \\
K-113 & 0.085 \\
\hline E-100 & 1.04 \\
E-101 & 0.22 \\
E-102 & 0.059 \\
E-103 & 0.24 \\
\hline
\end{tabular}


Table S10. Input parameters for annual sales (SSFB-based, TSFB-based, THSFB-1-based, and THSFB-2-based correspond to the production routes with SSFB, TSFB, THSFB-1, and THSFB-2 reactors chosen in unit(a), respectively)

\begin{tabular}{|c|c|c|c|c|c|}
\hline \multicolumn{2}{|r|}{ Items } & Values & Units & Notes & Sources \\
\hline \multicolumn{2}{|r|}{$P_{\text {dry }}$} & 15.48 & $10^{-5} \$ / \mathrm{kmol}$ & $\mathrm{a}$ & 8 \\
\hline \multicolumn{2}{|r|}{$P_{\text {ben }}$} & 1046.18 & $\$ / t$ & $\mathrm{~b}$ & 9 \\
\hline \multicolumn{2}{|r|}{$P_{\mathrm{tol}}$} & 1184.08 & $\$ / t$ & $\mathrm{~b}$ & 10 \\
\hline \multicolumn{2}{|r|}{$P_{\mathrm{xyl}}$} & 1270.72 & $\$ / \mathrm{t}$ & $\mathrm{b}$ & 11 \\
\hline \multicolumn{2}{|r|}{$P_{\text {hea }}$} & 938.60 & $\$ / \mathrm{t}$ & $\mathrm{b}$ & 12 \\
\hline \multirow{4}{*}{$M_{\mathrm{dry}}$} & SSFB-based & 2318 & $\mathrm{kmol} / \mathrm{h}$ & - & - \\
\hline & TSFB-based & 2153 & $\mathrm{kmol} / \mathrm{h}$ & - & - \\
\hline & THSFB-1-based & 1480 & $\mathrm{kmol} / \mathrm{h}$ & - & - \\
\hline & THSFB-2-based & 1540 & $\mathrm{kmol} / \mathrm{h}$ & - & - \\
\hline \multirow{4}{*}{$M_{\text {ben }}$} & SSFB-based & 2.54 & $\mathrm{t} / \mathrm{h}$ & - & - \\
\hline & TSFB-based & 4.15 & $\mathrm{t} / \mathrm{h}$ & - & - \\
\hline & THSFB-1-based & 4.98 & $\mathrm{t} / \mathrm{h}$ & - & - \\
\hline & THSFB-2-based & 8.86 & $\mathrm{t} / \mathrm{h}$ & - & - \\
\hline \multirow{4}{*}{$M_{\text {tol }}$} & SSFB-based & 20.82 & $\mathrm{t} / \mathrm{h}$ & - & - \\
\hline & TSFB-based & 22.20 & $\mathrm{t} / \mathrm{h}$ & - & - \\
\hline & THSFB-1-based & 23.06 & $\mathrm{t} / \mathrm{h}$ & - & - \\
\hline & THSFB-2-based & 26.28 & $\mathrm{t} / \mathrm{h}$ & - & - \\
\hline \multirow{4}{*}{$M_{\mathrm{xyl}}$} & SSFB-based & 21.45 & $\mathrm{t} / \mathrm{h}$ & - & - \\
\hline & TSFB-based & 21.08 & $\mathrm{t} / \mathrm{h}$ & - & - \\
\hline & THSFB-1-based & 25.64 & $\mathrm{t} / \mathrm{h}$ & - & - \\
\hline & THSFB-2-based & 20.67 & $\mathrm{t} / \mathrm{h}$ & - & - \\
\hline \multirow{4}{*}{$M_{\text {hea }}$} & SSFB-based & 5.48 & $\mathrm{t} / \mathrm{h}$ & - & - \\
\hline & TSFB-based & 5.09 & $\mathrm{t} / \mathrm{h}$ & - & - \\
\hline & THSFB-1-based & 11.91 & $\mathrm{t} / \mathrm{h}$ & - & - \\
\hline & THSFB-2-based & 7.79 & $\mathrm{t} / \mathrm{h}$ & - & - \\
\hline
\end{tabular}

a The dry gas price is gotten by the price of natural gas based on heat value.

${ }^{\mathrm{b}}$ The annual sales are calculated by the average prices of Chinese market in 2018. 
Table S11. Input parameters for total annual production costs

\begin{tabular}{cccc}
\hline Items & Values & Units & Sources \\
\hline$P_{\mathrm{met}}$ & 382.66 & $\$ / \mathrm{t}$ & 13 \\
$P_{\mathrm{LP}}$ & 2.00 & $10^{-6} \$ / \mathrm{kJ}$ & - \\
$P_{\mathrm{MP}}$ & 2.00 & $10^{-6} \$ / \mathrm{kJ}$ & - \\
$P_{\mathrm{HP}}$ & 3.00 & $10^{-6} \$ / \mathrm{kJ}$ & - \\
$P_{\mathrm{CW}}$ & 0.21 & $10^{-6} \$ / \mathrm{kJ}$ & - \\
$P_{\mathrm{Re}}$ & 8.98 & $10^{-6} \$ / \mathrm{kJ}$ & - \\
$P_{\mathrm{El}}$ & 16.00 & $10^{-6} \$ / \mathrm{kJ}$ & - \\
$P_{\mathrm{HPG}}$ & 3.00 & $10^{-6} \$ / \mathrm{kJ}$ & - \\
$M_{\mathrm{met}}$ & 180.2 & $\mathrm{t} / \mathrm{h}$ & - \\
$N U$ & 25 & - & - \\
$S U$ & 12480 & $\$ /$ operator $/ \mathrm{yr}$ & - \\
$N A$ & 25 & - & - \\
$S A$ & 24960 & $\$ /$ operator $/ \mathrm{yr}$ & - \\
$N C$ & 25 & - & - \\
$S C$ & 31200 & $\$ /$ operator/yr & - \\
\hline
\end{tabular}

Table S12. The characterization factors for environmental analysis ${ }^{14}$

\begin{tabular}{ccc}
\hline Items & Values & Units \\
\hline$\varphi^{\mathrm{LP}}$ & $1.157 \times 10^{-5}$ & points $/ \mathrm{kJ}$ \\
$\varphi^{\mathrm{MP}}$ & $1.157 \times 10^{-5}$ & points $/ \mathrm{kJ}$ \\
$\varphi^{\mathrm{HP}}$ & $1.157 \times 10^{-5}$ & points $/ \mathrm{kJ}$ \\
$\varphi^{\mathrm{ele}}$ & $2.648 \times 10^{-5}$ & points $/ \mathrm{kJ}$ \\
$\varphi^{\mathrm{CO}_{2}}$ & $4.524 \times 10^{-2}$ & points $/ \mathrm{kg}$ \\
\hline
\end{tabular}




\section{References}

(1) Wang T, Tang XP, Huang XF, Qian WZ, Cui Y, Hui XY, Yang W, Wei F. Conversion of methanol to aromatics in fluidized bed reactor. Catal Today. 2014; 233: 8-13.

(2) Chen ZH, Hou YL, Song WL, Cai D, Yang YF, Cui Y, Qian WZ. High-yield production of aromatics from methanol using a temperature-shifting multi-stage fluidized bed reactor technology. Chem Eng J. 2019; 371: 639-646.

(3) Chen ZH, Hou YL, Yang YF, Cai DL, Song WL, Wang N, Qian WZ. A multi-stage fluidized bed strategy for the enhanced conversion of methanol into aromatics. Chem Eng Sci. 2019; 204: 1-8.

(4) Xu CE. The Technologies of Catalytic Reforming. Beijing: China Petrochemical Press, 2014; pp 952-958.

(5) HCBBS, https://bbs.hcbbs.com/thread-2220468-1-1.html (accessed Sept 10, 2018).

(6) Seider WD, Seader JD, Lewin DR, Widagdo S. Product and Process Design Principles: Synthesis, Analysis and Evaluation, 3rd ed. Hoboken, NJ: John Wiley \& Sons, 2009; pp 534-641.

(7) Heydt GT. The probabilistic evaluation of net present value of electric power distribution systems based on the Kaldor-Hicks compensation principle. IEEE Trans Power Syst. 2018; 33 (4): 4488-4495.

(8) OilChem, http://oil.oilchem.net/oil/NG.shtml (accessed Dec 01, 2018).

(9) PPI, http://ben.100ppi.com/ (accessed Nov 27, 2018).

(10) OilChem, http://chem.oilchem.net/chemical/toluene.shtml (accessed Nov 24, 2018).

(11) ChemCP, http://www.chemcp.com/news/201810/900678.asp (accessed Nov 28, 2018).

(12) OilChem, http://price.oilchem.net/dmPrice/listProduct.lz?pName=C9\&webFlag=1\&hndz=0 (accessed Nov 29, 2018).

(13) OilChem, http://chem.oilchem.net/chemical/methanol.shtml (accessed Nov 25, 2018).

(14) Ecoinvent Database Version 3.3; Ecoinvent Centre: Bern, Switzerland, 2016. 